\title{
Unscrambling Illusory Inhibition and Catalysis in Nanoparticle Electrochemistry: Experiment and Theory
}

\author{
Lifu Chen*, Enno Kätelhön*, and Richard G. Compton ${ }^{\dagger}$ \\ Department of Chemistry, Physical and Theoretical Chemistry Laboratory, Oxford \\ University, South Parks Road, Oxford, OX1 3QZ, United Kingdom
}

\begin{abstract}
Recent theoretical work (Applied Materials Today 15 (2019), pp. 139-144) has however demonstrated that the cyclic voltammetry of particle-modified electrodes bears a significant level of complexity: Altered mass transport inside the porous layer gives rise to effects that may falsely suggest positive or negative catalysis. This communication reports experimental work that validates this theory. Using the well-studied oxidation of ferrocenemethanol at glassy carbon, we describe experiments in the presence of non-conducting electrochemically-inert microspheres that reproduce trends in the theoretical predictions and illustrate how experimental results may mislead. In addition, we present experimental data of the reduction of oxygen at glassy carbon electrodes modified with Nafion microspheres and show that, unless compared with theoretical work, an electrocatalytic activity of Nafion may falsely be inferred.
\end{abstract}

Keywords: Electrocatalysis, Nanoparticles, Nanoporous Electrodes,

*Authors contributed equally.

${ }^{\dagger}$ Corresponding author: Richard G. Compton, richard.compton@chem.ox.ac.uk 


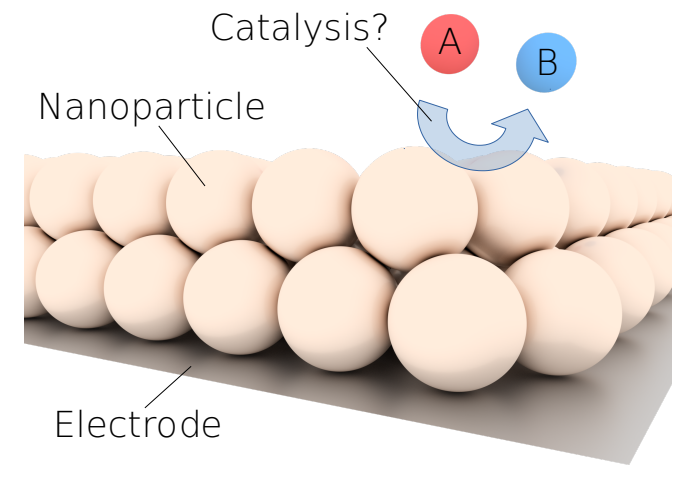

Graphical abstract

Nanoparticle-modified electrodes remain at the heart of electrocatalytic research and still continue to be one main focus in catalysis research. A recent communication in this journal [1] has provided an overview of related experimental and theoretical work, to which we here point the interested reader. Notable review papers include $[2,3,4,5,6,7,8]$ and offer excellent introductions to the topic.

In the analysis of the electrocatalytic properties of nanoparticles, cyclic voltammetry very commonly plays a central role. Materials of interest are deposited on an electrode surface and characterised in the presence of a suitable reactant. The voltammetry of the modified electrode is subsequently compared with its bare equivalent where an 'earlier' peak potential or a higher peak current might intuitively [9] suggest a catalytic activity of the nanoparticles. Recent theoretical work [1] has however led to the insight that, counter-intuitively, such experiments bear a significant level of complexity: Due to the geometrical constrains imposed on diffusion inside the porous layer formed by the nanoparticles, accelerated mass transport can occur locally where more pronounced concentration gradients are formed. Simultaneously, the mass transport towards the electrode may be retarded in other regions by the presence of the nanoparticles. As a result and in comparison to bare electrodes, porous electrodes may exhibit shifts in peak positions in any direction - anodic or cathodic - and peak currents may vary in a complex fashion. As this altered voltammetry is crucially determined by the electrode geometry and other experimental conditions, any analysis of such experimental data remains ambiguous unless accompanied by rigorous theoretical work. 
This communication reports experimental work that supports the theory mentioned above: Using the well-characterised one-electron oxidation of ferrocenemethanol in aqueous solution at glassy carbon, we show that voltammetric peak-to-peak separations and -peak currents follow theoreticallypredicted trends if electrodes are modified with carboxylated polystyrene microspheres which are non-conducting, non-reactive, and non-absorbing under the set experimental conditions. In addition, we illustrate the complexity of such analysis at the example of an often-studied reaction, the reduction of oxygen: A glassy carbon electrode decorated with a layer or layers of Nafion particles shows elevated voltammetric peak currents and peak potentials which, in the absence of any catalytic reaction, may falsely suggest electrocatalytic activity.

We employ a theoretical model detailed previously [1]. In short, a planar electrode is modified with inactive particles which are identical, spherical, and arrange in the hexagonal packing shown in Figure 1a. The resulting electrode geometry is then simplified along the lines of the diffusion-domain approximation [10] as illustrated in Figure 1b. Furthermore and in addition to the assumptions made in [1], we set the radius of the cylindrical diffusion domain $r_{\max }$, see Figure 1c, in a way that the packing density of the first layer of spheres in the original geometry matches the respective packing density in the diffusion domain. Please see supporting material for further details and derivations which demonstrate that this is the case when:

$$
r_{\max }=1.0501 r_{p}
$$

where $r_{p}$ is the particle radius. The electrochemical reaction is implemented as a reversible one-electron processes with the oxidised and the reduced species featuring equal diffusion coefficients. Bespoke simulation code has been developed which, despite some improvements, is similar to the code used in [1] and comprises large parts of it. Software testing [11] is of paramount importance in the development of electrochemical simulations and have conducted a broad range of tests including an analysis of the conservation of mass, comparisons with theoretical peak currents and peak-to-peak separations where feasible, and convergence studies.

Our analysis commences with an investigation of the electrochemically reversible ${ }^{3}$ reaction of ferrocenemethanol, $\mathrm{FcCH}_{2} \mathrm{OH}$, at particle-modified

\footnotetext{
${ }^{3}$ Please see below discussion and supporting material for a detailed analysis.
} 

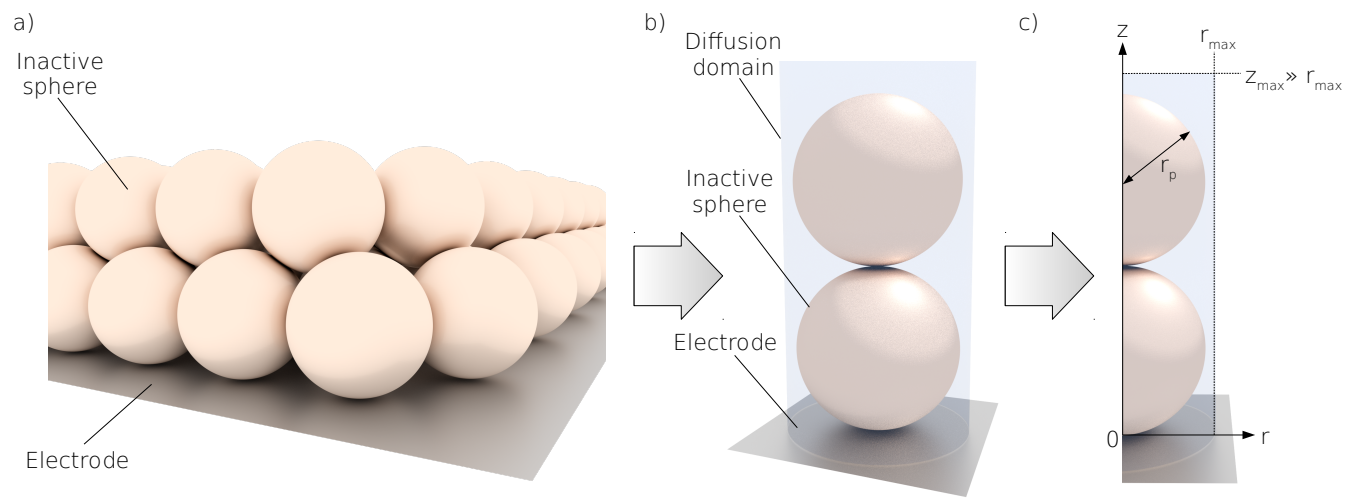

Figure 1: Illustration of the geometry simplified along the lines of the diffusion domain approximation. The figure has been adapted from [1].

glassy carbon electrodes. To this end, inactive carboxylated polystyrene microspheres $^{4}$ of equal sizes $\left(r_{p}=225 \mathrm{~nm}, 2.25 \mu \mathrm{m}\right.$, and $\left.10 \mu \mathrm{m}\right)$ are dropcast onto the electrode surface to form dense layers in a homogeneous hexagonal packing pattern as evident from the micrographs of the $10 \mu \mathrm{m}$ spheres shown in Figure 2. Corresponding micrographs for an electrode modified with smaller spheres featuring a radius of $2.25 \mu \mathrm{m}$ can be found in the supporting material and reveal that these arrange in a less symmetrical fashion. A comparison with Figure 1a nonetheless reveals that the electrode geometry for both particle sizes closely resembles the theoretical model.

Voltammograms are recorded at different scan rates at a bare electrode and at the electrodes modified with carboxylated polystyrene microspheres. The voltammetry of the bare electrodes is subsequently compared with Randles-ŠSevčík equation and the theoretically predicted peak-to-peak separation of $2.218 \frac{R T}{F}$ [12] which both apply for a reversible one-electron reaction. Good agreement is seen between experiment and theory as shown in the supporting material and the theoretical model is hence confirmed. On the basis of Randles-ŠSevč́k equation the diffusion coefficient is determined to $7.6 \cdot 10^{-10} \mathrm{~m}^{2} \mathrm{~s}^{-1}$ which matches the value of $(7.6 \pm 0.4) \cdot 10^{-10} \mathrm{~m}^{2} \mathrm{~s}^{-1}$

\footnotetext{
${ }^{4}$ Polystyrene is a good electrical insulator and hence electrochemically inactive if used as an electrode material at a liquid-solid interface. The stability of the microspheres is investigated in the supporting material: No changes can be seen on micrographs of the deposited spheres before and after an electrochemical experiment.
} 

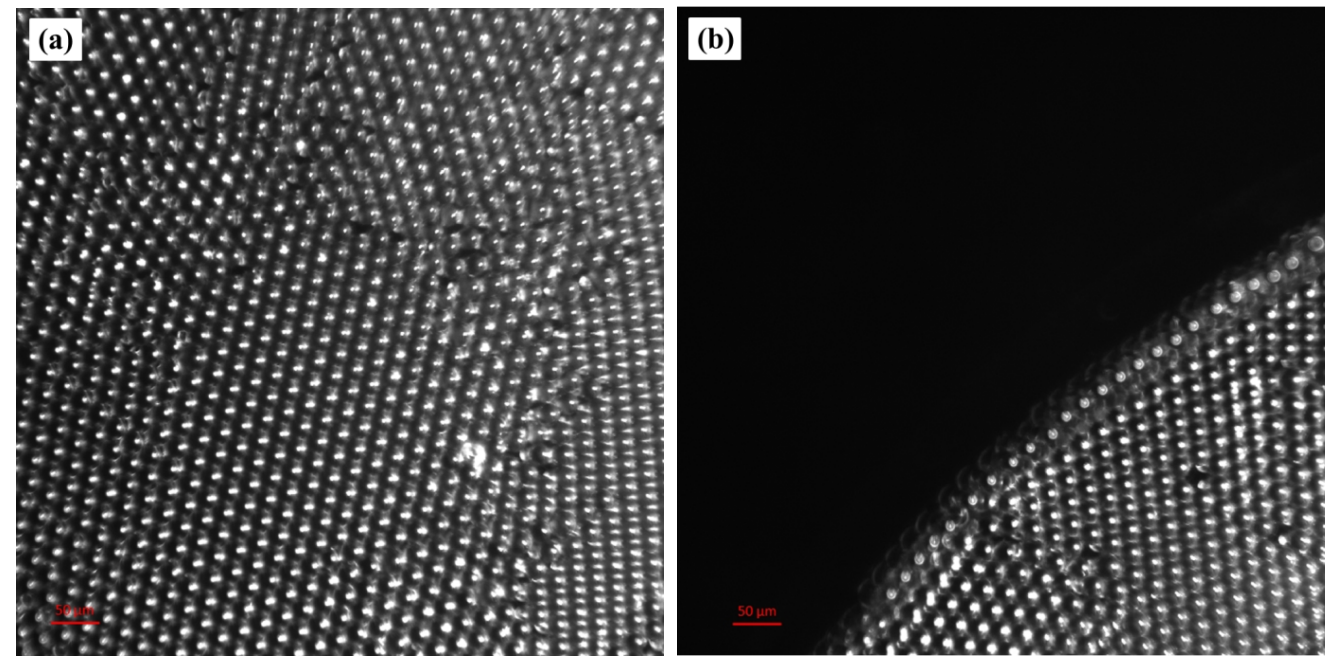

Figure 2: Micrographs of polybead microspheres dropcast on a glassy carbon electrode. Microspheres feature a radius of $10 \mu \mathrm{m}$ and are deposited in one layer. (a) middle view of the electrode (b) edge view of the electrode.

priorly reported [13]. The formal potential is found to be $0.19 \mathrm{~V}$ vs SCE via determination of the mid-point potential.

Moving on to modified electrodes, Figure 3 presents aggregated data of large number of voltammograms recorded for an average surface coverage of one layer of microspheres: The measured peak-to-peak separations (dashed lines) are plotted as a function of scan rate for comparison with simulated data (solid lines). Following the theoretical prediction, we find significant variations in peak-to-peak separations for all sphere radii. Most notably and depending on the sphere radius, separations may increase or decrease with increasing scan rates which may falsely suggest negative or positive catalysis, respectively. Simulated trends are reproduced and, for the smallest spheres, an almost an ideal match between experiment and theory is seen. Differences may relate to the assumptions made in the diffusion domain approximation, imperfections in the deposited layers of spheres, and a potentially limited electrolytic support within nano-scaled features inside the porous layer. Similar results where obtained for two and three layers of spheres as shown in the supporting material.

Having shown the applicability of the theoretical model in terms of peakto-peak separations, our analysis turns towards measured peak currents. Figure 4 plots the peak currents as a function of the scan rate for an average 


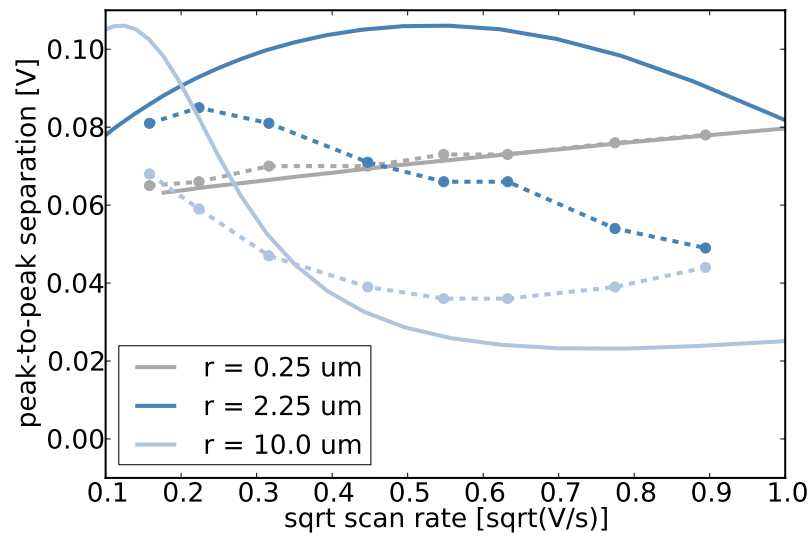

Figure 3: Comparison of measured (dashed lines) and simulated (solid lines) peak-to-peak separations as a function of scan rate for three different sizes of polybead microspheres and an average surface coverage of one layer of spheres. All simulations here and in the following use a diffusion coefficient of $7.61 \times 10^{-10} \mathrm{~m}^{2} \mathrm{~s}^{-1}$. Experimental data was recorded in a solution comprising $1 \mathrm{mM}$ ferrocenemethanol and $100 \mathrm{mM}$ potassium chloride. Please see supporting material for further experimental details.

surface coverage of one layer of spheres (dashed lines). In agreement with theoretical predictions and in stark contrast to the expected square-root dependency of the peak current on the scan rate at a bare electrode, significant variations are seen with the scan rate and the particle size. The same figure compares experimental data with simulations (solid lines) which reproduce trends in experimental data and support the validity of the theoretical model. Similar results are obtained for averages of two and three layers are shown in the supporting material.

The above experimental data has confirmed the theoretically-predicted complexity of any voltammetric analysis at nano-structured surfaces. Using the relatively simple and well-studied reaction of ferrocenemethanol at glassy carbon, we have demonstrated that a sole voltammetric analysis of particlemodified electrode cannot provide insight into the catalytic properties of the material deposited unless accompanied by suitable theoretical models or data obtained from other experimental techniques. To further emphasise this fact, the following presents data of a reaction that is much more in the focus of 


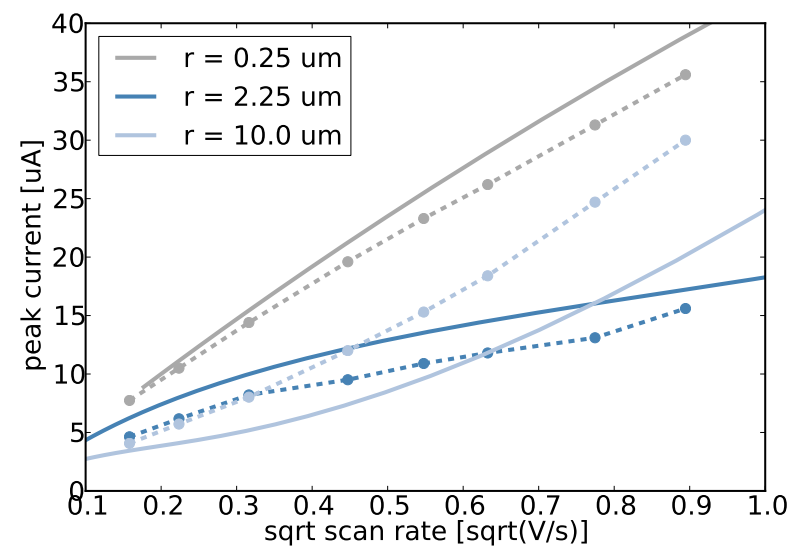

Figure 4: Comparison of measured (dashed lines) and simulated (solid lines) peak currents as a function of scan rate for three different sizes of polybead microspheres. Experimental data was collected from the same voltammograms analysed in Figure 3.

current research, namely the reduction of oxygen ${ }^{5}$. In comparison to ferrocenemethanol, this is a much more complex process as it involves multiple electron-transfers, reaction intermediates, and species that may adsorb at some electrode surfaces:

$$
\mathrm{O}_{2}+2 \mathrm{H}^{+}+2 e \longrightarrow \mathrm{H}_{2} \mathrm{O}_{2}
$$

or:

$$
\mathrm{O}_{2}+4 \mathrm{H}^{+}+4 e \longrightarrow 2 \mathrm{H}_{2} \mathrm{O}
$$

While the above theoretical model of a reversible one-electron transfer is hence not applicable, experimental data of the oxygen reduction nonetheless strikingly illustrates the complexity of the mass transport in porous layers and points out the risk of misinterpretation of experimental results.

The following investigates the voltammetry of a glassy carbon electrode modified with layers of Nafion particles $[15,16]$ featuring an average radius of $430 \mathrm{~nm}$. To this end, we first measure the electrochemical activity of

\footnotetext{
${ }^{5}$ Please see the review [14] by Shao et al. for a comprehensive discussion of recent research activities related to proton exchange membrane fuel cells.
} 
the Nafion particles: Voltammograms are recorded at a bare- and a modified electrode in Argon-saturated phosphate buffered saline solution at $\mathrm{pH}$ 7.4 where no changes are seen except for an expected [17] small change in the electrode capacity, see supporting material, suggesting the particles are inactive in the buffer solution.

The bare glassy carbon electrode is subsequently characterised in airsaturated phosphate buffered saline solution at $\mathrm{pH} 7.4$ featuring an oxygen concentration of $0.26 \mathrm{mM}$ [18], see supporting material, which is known to be the two-electron process shown in Equation 2 [19]. Via a Tafel analysis [20], the electrochemical transfer coefficient [21, 22] is determined to 0.25 and by comparison with the multiple-electron-transfer irreversible RandlesŠevčík the diffusion coefficient is found to be $2.00 \cdot 10^{-9} \mathrm{~m}^{2} \mathrm{~s}^{-1}$. Both values are in excellent agreement with prior literature values of $0.24 \pm 0.06$ [23] and $1.95 \cdot 10^{-9} \mathrm{~m}^{2} \mathrm{~s}^{-1}$ ) [24], respectively. Figure 5 compares the voltammetry of a bare glassy carbon electrode and particle-modified electrodes. The latter are modified with the above Nafion particles while various average numbers of particle layers are deposited on the electrode surface. As one would anticipate from the analysis of the voltammetry of ferrocene methanol at similar electrodes, the figure reveals a strong dependency of the voltammograms on the amount of Nafion deposited. Most notably, there is a shift of about 60 $\mathrm{mV}$ in the position of the oxygen peak towards lower overpotentials and a dramatic increase of more than $40 \%$ in the peak current. The Tafel analysis further yields an $\alpha$ value of 0.39 rather than 0.25 at a bare glassy carbon electrode, see supporting material. All three findings might intuitively be attributed to a false and electrochemically implausible electrocatalytic activity of Nafion particles, though the above theoretical analysis suggests they are likely purely diffusional effects.

In conclusion, this work has demonstrated the paramount importance of theoretical modelling in all electrocatalytic analysis of nanoparticles on an electrode surface. Prior theoretical work is validated which has suggested that, under most common experimental conditions, the voltammetry of particle-modified electrodes features an unexpected level of complexity: Peak positions and -currents are altered dramatically, a straightforward analysis is obstructed, and there is a risk of false interpretation in the absence of theoretical modelling. Using ferrocenemethanol at glassy carbon electrodes as a model system, we find good agreement of theoretically predicted trends and measured data. In addition, the complexity of the analysis of more complex reactions such as the reduction of oxygen is demonstrated 


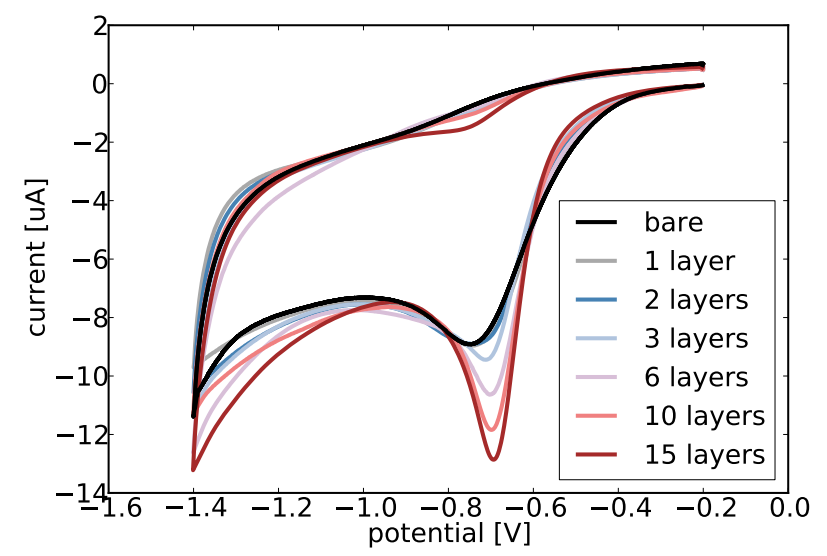

Figure 5: Voltammetry of air-saturated phosphate-buffered saline solution at a glassy carbon electrode modified with Nafion particles. Particles feature a radius of $430 \mathrm{~nm}$ and have been dropcast onto the electrode. Voltammograms are recorded at a scan rate of $100 \mathrm{mV} \mathrm{s}^{-1}$. Please see supporting material for full experimental details.

experimentally and risks of misinterpreting experimental results are pointed out.

\section{Acknowledgement}

We thank Mr Ruochen Xie for help with the microscopy.

\section{Data Availability}

The raw/processed data required to reproduce these findings cannot be shared at this time due to technical or time limitations.

\section{References}

[1] E. Kätelhön, L. Chen, R. G. Compton, Nanoparticle electrocatalysis: Unscrambling illusory inhibition and catalysis, Applied Materials Today (2019) $139-144$.

[2] R. G. Compton, G. G. Wildgoose, N. V. Rees, I. Streeter, R. Baron, Design, fabrication, characterisation and application of nanoelectrode arrays, Chemical Physics Letters 459 (2008) 1 - 17. 
[3] R. W. Murray, Nanoelectrochemistry: Metal nanoparticles, nanoelectrodes, and nanopores, Chemical Reviews 108 (2008) 2688-2720.

[4] Q. Lu, J. G. Chen, J. Q. Xiao, Nanostructured electrodes for highperformance pseudocapacitors, Angewandte Chemie International Edition 52 (2013) 1882-1889.

[5] C. Batchelor-McAuley, E. Kätelhön, E. O. Barnes, R. G. Compton, E. Laborda, A. Molina, Recent advances in voltammetry, ChemistryOpen 4 (2015) 224-260.

[6] F. Wang, X. Wu, C. Li, Y. Zhu, L. Fu, Y. Wu, X. Liu, Nanostructured positive electrode materials for post-lithium ion batteries, Energy Environmental Science 9 (2016) 3570-3611.

[7] K. J. Aoki, Voltammetry at a single nano-electrode by varying electrode diameters: Review, Journal of Electroanalytical Chemistry 779 (2016) $7-17$.

[8] L. Zhang, Z.-J. Zhao, J. Gong, Nanostructured materials for heterogeneous electrocatalytic $\mathrm{CO}_{2}$ reduction and their related reaction mechanisms, Angewandte Chemie International Edition 56 (2017) 1132611353.

[9] E. Härk, R. Jäger, P. E. Kasatkin, P. Möller, R. Kanarbik, I. Tallo, U. Joost, J. Aruväli, P. Paiste, H. Jiang, T. Kallio, K. Kirsimäe, E. Lust, The electrochemical activity of two binary alloy catalysts toward oxygen reduction reaction in $0.1 \mathrm{M} \mathrm{KOH}$, Journal of Solid State Electrochemistry 22 (2018) 31-40.

[10] R. G. Compton, E. Laborda, K. R. Ward, Understanding Voltammetry: Simulation of Electrode Processes, World Scientific Publishing Company, 2013.

[11] E. Kätelhön, R. G. Compton, Testing and validating electroanalytical simulations, Analyst 140 (2015) 2592-2598.

[12] R. G. Compton, C. E. Banks, Understanding Voltammetry (3rd Edition), World Scientific Press, 2018. 
[13] C. Amatore, N. Da Mota, C. Sella, L. Thouin, Theory and Experiments of Transport at Channel Microband Electrodes under Laminar Flows. 1. Steady-State Regimes at a Single Electrode, Analytical Chemistry 79 (2007) 8502-8510.

[14] M. Shao, Q. Chang, J.-P. Dodelet, R. Chenitz, Recent Advances in Electrocatalysts for Oxygen Reduction Reaction, Chemical Reviews 116 (2016) 3594-3657.

[15] H. Yang, X. Li, C. Batchelor-McAuley, S. V. Sokolov, R. G. Compton, Nafion particles doped with methyl viologen: electrochemistry, Physical Chemistry Chemical Physics 20 (2017) 682-689.

[16] L. Chen, C. Lin, R. G. Compton, Electrochemical characterisation and comparison of transport in Nafion films and particles, Physical Chemistry Chemical Physics 21 (2019) 607-616.

[17] F. Lufrano, P. Staiti, M. Minutoli, Influence of Nafion Content in Electrodes on Performance of Carbon Supercapacitors, Journal of The Electrochemical Society 151 (2004) A64-A68.

[18] R. E. Davis, G. L. Horvath, C. W. Tobias, The solubility and diffusion coefficient of oxygen in potassium hydroxide solutions, Electrochimica Acta 12 (1967) 287-297.

[19] K. M. Sundberg, W. H. Smyrl, Lj. Atanasoska, R. Atanasoski, Surface Modification and Oxygen Reduction on Glassy Carbon in Chloride Media, Journal of The Electrochemical Society 136 (1989) 434-439.

[20] D. Li, C. Lin, C. Batchelor-McAuley, L. Chen, R. G. Compton, Tafel analysis in practice, Journal of Electroanalytical Chemistry 826 (2018) $117-124$.

[21] R. Guidelli, R. G. Compton, J. M. Feliu, E. Gileadi, J. Lipkowski, W. Schmickler, S. Trasatti, Defining the transfer coefficient in electrochemistry: An assessment (IUPAC Technical Report), Pure and Applied Chemistry 86 (2014) 245-258.

[22] R. Guidelli, R. G. Compton, J. M. Feliu, E. Gileadi, J. Lipkowski, W. Schmickler, S. Trasatti, Definition of the transfer coefficient in 
electrochemistry (IUPAC Recommendations 2014), Pure and Applied Chemistry 86 (2014) 259-262.

[23] R. Nissim, R. G. Compton, Nonenzymatic Electrochemical Superoxide Sensor, ChemElectroChem 1 (4) (2014) 763-771.

[24] L.-K. Ju, C. S. Ho, Measuring oxygen diffusion coefficients with polarographic oxygen electrodes: I. Electrolyte solutions, Biotechnology and Bioengineering 27 (1985) 1495-1499. 